\title{
Virtually Extended Surgical Drilling Device: Virtual Mirror for Navigated Spine Surgery
}

\author{
Christoph Bichlmeier ${ }^{1}$, Sandro Michael Heining ${ }^{2}$, \\ Mohammad Rustaee ${ }^{1}$, and Nassir Navab ${ }^{1}$ \\ ${ }^{1}$ Computer Aided Medical Procedures (CAMP), TUM, Munich, Germany \\ \{bichlmei, navab\}@cs.tum.edu, \{mohammad.rustaee\}@gmail.com \\ 2 Trauma Surgery Department, Klinikum Innenstadt, LMU, Munich, Germany \\ \{Sandro-Michael. Heining\} @med . uni-muenchen. de
}

\begin{abstract}
This paper introduces a new method for navigated spine surgery using a stereoscopic video see-through head-mounted display (HMD) and an optical tracking system. Vertebrae are segmented from volumetric CT data and visualized in-situ. A surgical drilling device is virtually extended with a mirror for intuitive planning of the drill canal, control of drill direction and insertion depth. The first designated application for the virtually extended drilling device is the preparation of canals for pedicle screw implantation in spine surgery. The objective of surgery is to install an internal fixateur for stabilization of injured vertebrae. We invited five surgeons of our partner clinic to test the system with realistic replica of lumbar vertebrae and compared the new approach with the classical, monitor-based navigation system providing three orthogonal slice views on the operation site. We measured time of procedure and scanned the drilled vertebrae with $\mathrm{CT}$ to verify accuracy of drilling.
\end{abstract}

\section{Introduction}

Implantation of pedicle screws is a frequently performed procedure in spine surgery. The pedicle approach is not only used to perform minimally invasive spinal interventions like vertebroplasty and kyphoplasty in osteoporotic fracture conditions but also in most dorsal stabilization procedures. The region around an injured vertebra is stabilized with an internal fixateur attached to intact vertebrae with screws drilled into their pedicles. Drilling canals into intact pedicles around injured vertebrae is an essential, preparative procedure for implantation of pedicle screws. The surgical task of pedicle screw placement in the lumbar and thoracic spine remains interesting even after a decade of image-guided surgery in the spine, which has lead to a variety of computer-aided techniques using different imaging modalities [1]: Basic techniques developed from using anatomic descriptions of the entry points in different spinal levels and the typical directions for pedicle screws ("droit devant" [2]) and static x-ray control after instrumentation to intra-operative 2D-flouroscopic control; advanced techniques from CT-based surgical navigation to intra-operative Iso-C-3D-control. 
Regarding this procedure, state-of-the-art navigation systems consist of an optical tracking system that locates surgical instruments and the patient at the operation site. Imaging data is presented with three orthogonal slice views on the operation site on an external monitor. Position of slices follows the drill, this means the intersection line is aligned with the drill axes. In addition some systems provide a $3 \mathrm{D}$ visualization of the region around the operation site using polygonal surfaces or direct volume rendering.

Augmented Reality (AR) for intra-operative visualization and navigation has been a subject of intensive research and development during the last decade [3] . Azar et al. presented a user performance analysis with four different navigation systems for needle placement. Results show that a HMD based system performs "better in avoiding the surrounding structures"' and needle procedures perform "in a shorter amount of time" [5]. Traub et al. presented a clinical evaluation of different visualization modes for surgical drilling [6]. Perceptive advantages of the virtual mirror were first described in [7. Later the laparoscopic virtual mirror was introduced for liver resection [8]. Thanks to a virtual mirror using a stereoscopic video see-through head-mounted display (HMD) and an optical tracking system (see figure 1(b)], we introduce a new computer aided system for drilling in navigated spine surgery. Due to in-situ visualization (2.2) mental mapping of medical imagery, which is presented on radiographs or on a monitor within in the operating room (OR), to the patient is not necessary. The visualization can be directly registered with the real operation site for diagnoses, surgical planning, and intraoperative navigation. Perception of 3D in-situ visualization is intuitive, however interaction with such $3 \mathrm{D}$ data is not practical. In a laboratory setting one can move around the virtually augmented scene to perceive layout, size and its relative position to further objects. Although not every desired view on the object is possible. Regarding a typical scenario in the OR, the surgeon is surrounded by clinical staff, equipment and the patient. A point of view on the operation site for instance from beneath the operation table is impossible. Here, the virtual mirror assists to enable intuitively the desired perspectives.

\section{Method}

The following section provides a detailed description of the AR system, the setup of the clinical study and the integration of the virtual mirror.

\subsection{AR System}

For superior registration quality the system uses two synchronized tracking systems. The single camera inside-out tracking system allows for a high rotational precision 9] necessary for tracking the stereoscopic video see-through head mounted display (HMD) [10]. The optical outside-in tracking system from A.R.T GmbH (Weilheim, Germany) with four infrared cameras fixed to the ceiling covers a large working area $(3 x 3 x 2 \mathrm{~m})$. Once this system is calibrated, it provides 
stable tracking data for a long term unless cameras are moved. The system is capable of tracking the targets in our setup with an accuracy of $<0.35[\mathrm{~mm}]$ RMS.

Both of the systems use the same kind of retro reflective fiducial markers offering a registration free transformation from one tracking system to the other. To recover the six degrees of freedom of a rigid body, the external optical tracking system requires at least four rigidly attached markers. Fiducial markers are attached to the surgical drill as well as to the phantom. The reference frame target has an exceptional function as it enables the transition between the insideout and the outside-in tracking systems. Both tracking systems calculate the same coordinate system for the reference frame. All targets are shown in figure 1(a) Further details of the used hardware and the tracking system is described in [7,11].

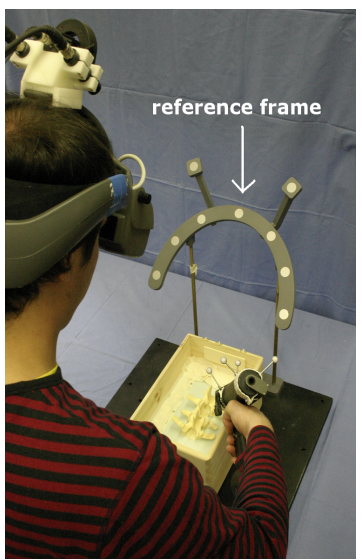

(a) View from outside.

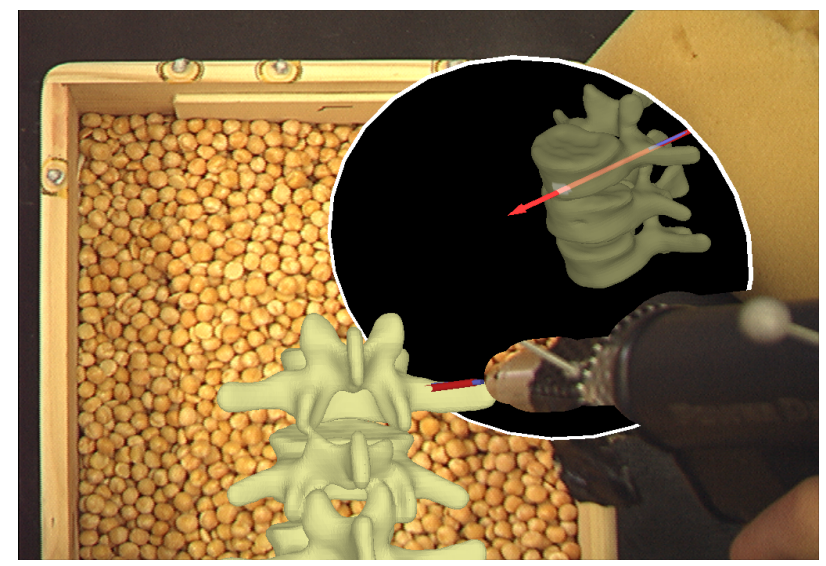

(b) Surgeon's view through HMD.

Fig. 1. Virtual and real objects of the AR scene. Tracked targets are surgical drilling device, phantom and the reference frame with 9 fiducial markers.

\subsection{In-Situ Visualization}

All augmentations on targets, which are tracked by the optical outside-in tracking system, have to be positioned respective the reference frame of the inside-out tracking system. The transformation for in-situ visualization can be described by ${ }^{C T} H_{\text {ref }}$.

$$
{ }^{C T} H_{\text {ref }}={ }^{C T} H_{\text {phantom }} *{ }^{\text {phantom }} H_{\text {ext }} *\left({ }^{\text {ref }} H_{\text {ext }}\right)^{-1}
$$

Fiducial markers are attached to the boundaries of the wooden box. The optical outside-in tracking system provides the transformations ${ }^{\text {phantom }} H_{\text {ext }}$ and ${ }^{r e f} H_{\text {ext }}$. Markers are segmented automatically from the volumetric imaging data. Correspondence of segmented positions and tracking data results in a registration matrix ${ }^{C T} H_{\text {phantom }}$ that aligns the data volume with the tracked object. 
Regarding a real surgical situation, data of an intraoperative CT scan with an optically tracked C-arm device can be registered with the patient and visualized 12 .

\subsection{Phantom}

For primary tests and prospective evaluation, we built a phantom consisting of three vertebrae embedded in a silicone mold. The outer two vertebrae are replaceable. The use of the silicone mold avoids multiple scans for every new experiment. Therefore we created further silicone molds (see figure 2(a) to reproduce the outer two vertebrae using synthetic two-component resin, which has similar physical properties as real vertebrae. According to the surgeons, material is slightly harder than real bone, which does not affect quality of drilling. Reproduced vertebrae fit precisely into the original silicon mold.

In a real scenario the surgeon only views a small area of vertebrae and therefore is able to roughly estimate the pose of the spinal column. Real pose of the spine can only be estimated. The silicone mold holding the three vertebrae was installed into a wooden box, which was filled with peas to simulate such restricted direct view in spine surgery (figure 2(b) .

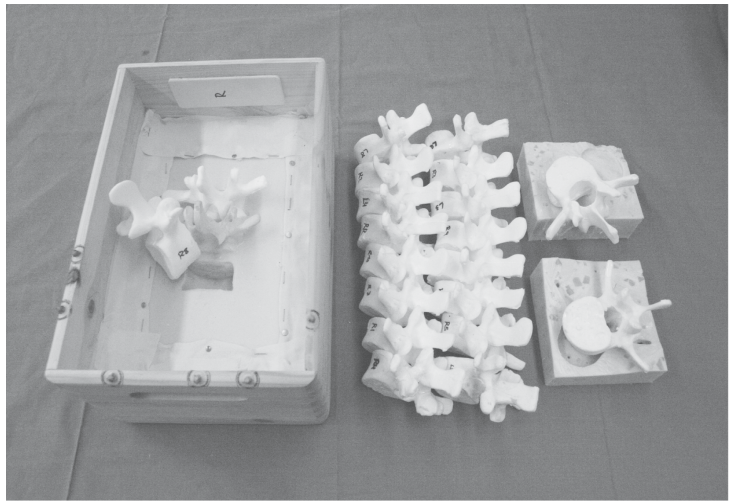

(a) Phantom, silicone mold and reproduced vertebrae.

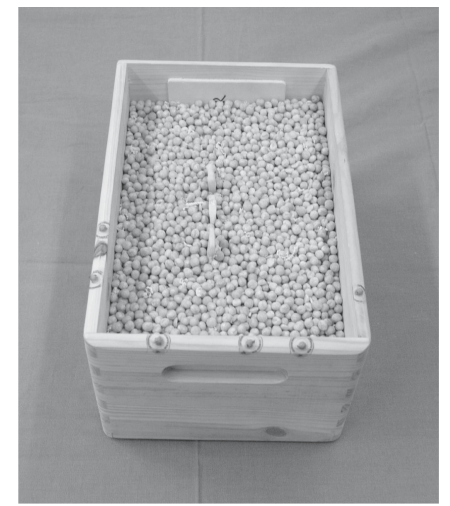

(b) Restricted view on vertebrae.

Fig. 2. Phantom for drilling experiments

\subsection{Integration of the Virtual Mirror}

We suggest dividing the procedure of drilling pedicles into two steps. First a drill canal is planed and defined, second the drilling itself is performed. Virtual components of the AR scene include a polygonal surface model of the vertebrae, a red arrow supporting the interactive planning of the drill canal, the virtual mirror and a blue cylinder representing the tracked drill. Depending on the position of objects, a virtual model of the drilling device is visible in the mirror 
image. Handling the problem of misleading depth perception is a major issue for AR systems. Virtual objects can only be presented superimposed on real objects. The model of the drilling device is used to create a depth mask with the stencil buffer 1 to provide occlusion, when the drilling device is positioned between the observer and visualization of segmented vertebrae or the virtual mirror.

Planning. The red arrow is initially orientated to the drill direction and positioned at its tip. The surgeon moves the drill to the visible entry point on the vertebrae and orientates the read arrow to the optimal drill canal. To ensure the correct position of the drill canal, the mirror is used to provide side views of the semi-transparent vertebrae. The mirror can be rotated on a circular path around the drill axes (radius $=10 \mathrm{~cm}$ ) by rotating the drilling device around its axes. For ease of use the rotation angle of the drilling device is multiplied by an adjustable factor to change the position of the mirror. This enables the surgeon to move the mirror around the target while only rotating the drill by small angle. Thus only slight motion of the drilling device provides all desired side views (see figures 34). When the canal is positioned correctly, it can be locked. It will then remain at a fixed position inside the vertebrae during the following steps of the procedure.

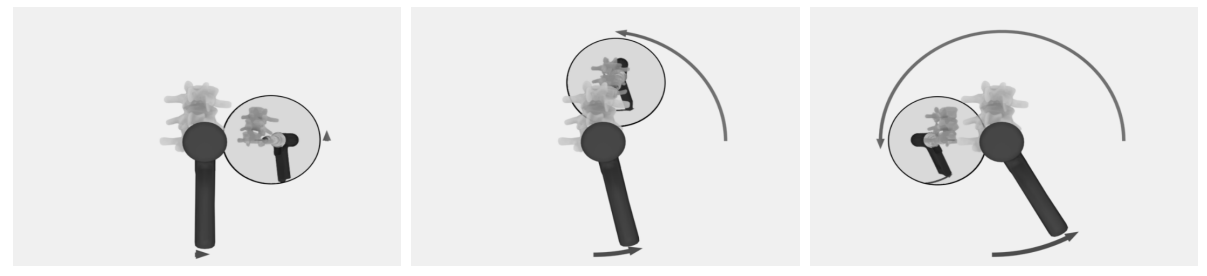

Fig. 3. Descriptive Illustration: Angle of mirror rotation around the drill tip(green/bright arrow) is the multiplied rotation angle of the drilling device around the drill axes (red/dark arrow)

Drilling. Once the canal is defined and locked the mirror automatically moves to a position in front of the drill, orthogonally to the drill direction. Now the surgeon moves the mirror to a desirable position that enables a view on the exit point on the bottom of the vertebrae but also allows for control of depth insertion. The mirror can be positioned automatically. However, we suggest to let the surgeon define its position according to the particular scenario, e.g. pose of the patient, position of equipment, surgeon and surgical staff during the operation and position of the operation site. When the ideal position for the mirror is found, it can be locked and remains respective the vertebrae.

A virtual spot light is attached to the drill tip and orientated to the drill direction. The non-realistic behavior of spot lights in OpenGL here turns into

${ }^{1}$ The stencil buffer is an additional buffer besides the color buffer and depth buffer found on modern computer graphics hardware. It can be used to limit the area of rendering. 
an advantage. Spot lights are not blocked by surfaces. Even surfaces not visible for the light source are illuminated, if they are located inside the cone of the spot light. Therefore the spot light illuminates the entry point at the pedicle as well as the exit point on the opposite side of the vertebrae, which is visible only through the mirror (see figure 1(b)]. Regarding the surgical task, the drill has to be aligned with the defined drill canal at the entry point using visual cues due to the spot light and intersection of drill, vertebrae and drill canal. Thereupon the drill has to be reoriented until the visible spot light is aligned with the exit point on the back of the vertebrae seen through the mirror.
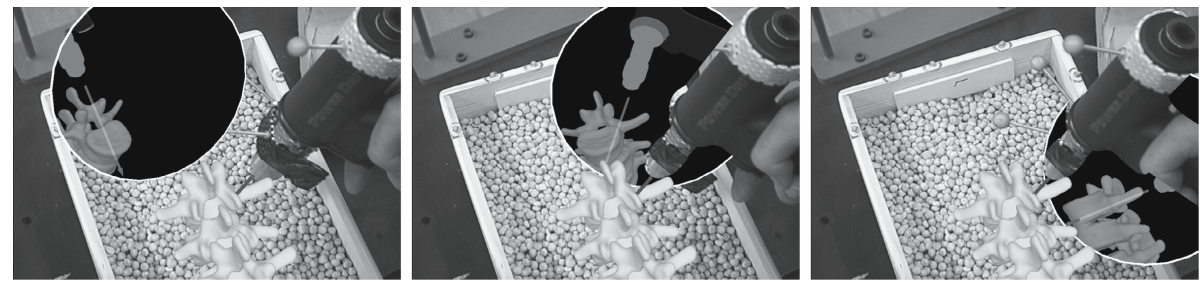

Fig. 4. Mirror is rotated around the operation site to check the position of the drill canal. Box was filled with peas to simulate the restricted view on the operation site.

\section{Results}

We asked five surgeons to drill preparative canals $\left(\varnothing_{\text {drill }}=4 \mathrm{~mm}\right)$ for pedicle screw implantation into the two replaceable lumbar vertebrae described in section 2.3. We then compared a classical monitor based navigation system with the present method. Regarding navigated drilling for pedicle screw implantation, three of the surgeons were highly experienced, one had low experience and the last one had no experience. Furthermore all of them had been exposed to our system within the scope of a different evaluation study. In real surgery the surgeons prepare the pedicles to avoid gliding off and injuring anatomy before they start drilling. In this experiment, however the surgeons start drilling directly into bones. Each subject has to consecutively drill four canals using each method. Overall, we analyzed quality of 20 canals for each method and measured time of the procedure. Regarding the duration of drilling one canal, using the classical monitor based method is faster than the proposed method. This time lag is due to the fact that the present method requires a planning phase. This is not intended for the classical navigation system. However when comparing the quality of drill canals our method proves to be more accurate(see table 1).

Scale of accuracy was defined as $1=$ perfect, $2=$ acceptable and $3=$ perforation [13. According to the surgeons the planning step enables a final check of canal position and allows for collaborative decision making before the vertebra is drilled. 
Table 1. Tendency of measured data shows higher accuracy using the virtual mirror/HMD based method

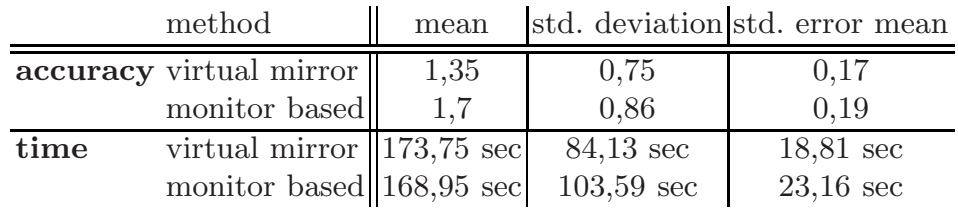

\section{Discussion}

Regarding evaluation of the system, we know that experimenting with five surgeons, each drilling only 8 canals, is not enough for drawing statistically valid conclusions, however because of the limited accessibility of the surgeons such experiments need to be completed in the future. We also plan to test different materials for artificial vertebrae. In the current system the surgeon needs to wear the HMD, however we developed a HMD hanging from the ceiling, which removes the discomfort due to the weight.

\section{Conclusion}

This paper introduces a new method for navigated spine surgery using an augmented reality system. Medical imaging data is visualized and presented with a stereoscopic video see-through HMD. The virtual part of the AR scene is extended by a virtual mirror to support more intuitive visualization and more accurate navigation. Furthermore five trauma surgeons tested the method with an experimental setup. Analysis of measured data proves the system to be promising both in terms of accuracy and usability.

\section{Acknowledgments}

We would like to thank Frank Sauer, Ali Khamene from Siemens Corporate Research (SCR) for placing the HMD system at our disposal. Thanks to A.R.T. $\mathrm{GmbH}$ for providing the outside-in tracking system. We also want to thank the radiologists and surgeons of Klinikum Innenstadt München for their precious contribution in obtaining medical data and evaluating our system. Thanks also to Joerg Traub, Marco Feuerstein and Tobias Sielhorst for their support.

\section{References}

1. Hart, R.A., Hansen, B.L., Shea, M., Hsu, F., Anderson, G.J.: Pedicle screw placement in the thoracic spine: a comparison of image-guided and manual techniques in cadavers. spine 30, 326-331 (2005)

2. Roy-Camille, R., Saillant, G., Mazel, C.: Internal fixation of the lumbar spine with pedicle screw plating. Clin. Orthop. 203, 7-17 (1986) 
3. Birkfellner, W., Figl, M., Huber, K., Watzinger, F., Wanschitz, F., Hummel, J., Hanel, R., Greimel, W., Homolka, P., Ewers, R., Bergmann, H.: A head-mounted operating binocular for augmented reality visualization in medicine - design and initial evaluation. TMI 21(8), 991-997 (2002)

4. King, A., Edwards, P., Maurer Jr., C., de Cunha, D., Gaston, R., Clarkson, M., Hill, D., Hawkes, D., Fenlon, M., Strong, A., Cox, T., Gleeson, M.: Stereo augmented reality in the surgical microscope. PresTVE 9(4), 360-368 (2000)

5. Azar, F.S., Perrin, N., Khamene, A., Vogt, S., Sauer, F.: User performance analysis of different image-based navigation systems for needle placement procedures. Proceedings of SPIE. 5367, 110-121 (2004)

6. Traub, J., Stefan, P., Heining, S.M.M., Tobias Sielhorst, C.R., Euler, E., Navab, N.: Hybrid navigation interface for orthopedic and trauma surgery. In: Larsen, R., Nielsen, M., Sporring, J. (eds.) MICCAI 2006. LNCS, vol. 4190, pp. 373-380. Springer, Heidelberg (2006)

7. Bichlmeier, C., Sielhorst, T., Navab, N.: The tangible virtual mirror: New visualization paradigm for navigated surgery. In: AMIARCS - The Tangible Virtual Mirror: New Visualization Paradigm for Navigated Surgery, Copenhagen, Denmark, MICCAI Society (2006)

8. Navab, N., Feuerstein, M., Bichlmeier, C.: Laparoscopic virtual mirror - new interaction paradigm for monitor based augmented reality. In: Virtual Reality, Charlotte, North Carolina, USA, March 2007, pp. 43-50 (2007)

9. Hoff, W.A., Vincent, T.L.: Analysis of head pose accuracy in augmented reality. IEEE Trans. Visualization and Computer Graphics 6 (2000)

10. Sauer, F., Schoepf, U.J., Khamene, A., Vogt, S., Das, M., Silverman, S.G.: Augmented reality system for ct-guided interventions: System description and initial phantom trials. Medical Imaging: Visualization, Image-Guided Procedures, and Display 5029, 384-394 (2003)

11. Sauer, F., Khamene, A., Bascle, B., Rubino, G.J.: A head-mounted display system for augmented reality image guidance: Towards clinical evaluation for imri-guided neurosurgery. In: MICCAI, London, UK, pp. 707-716. Springer, Heidelberg (2001)

12. Feuerstein, M., Mussack, T., Heining, S.M., Navab, N.: Registration-free laparoscope augmentation for intra-operative liver resection planning. In: SPIE Medical Imaging, San Diego, California, USA, vol. 203, pp. 7-17 (2007)

13. Arand, M., Schempf, M., Hebold, D., Teller, S., Kinzl, L., Gebhard, F.: Precision of navigation-assisted surgery of the thoracic and lumbar spine. Unfallchir. 106, 899-906 (2003) 